Original Research Paper

\title{
On the Comparison of Methods of Estimating Missing Values in Rectangular Lattice Designs
}

\author{
Abimibola V. Oladugba, Emmanuel O. Ossai and Tobias E. Ugah \\ Department of Statistics, University of Nigeria, Nsukka, Nigeria
}

Article history

Received: 25-05-2018

Revised: $18-07-2018$

Accepted: 27-07-2018

Corresponding Author:

Abimibola V. Oladugba

Department of Statistics,

University of Nigeria, Nsukka, Nigeria

Email: abimibola.oladugba@unn.edu.ng

\begin{abstract}
Missing values occur in almost all research which lead to ambiguity in data analysis. It becomes necessary that appropriate consideration is made in order to provide an efficient and valid analysis. Researchers have developed and compared a variety of methods of estimating missing values in experimental designs; however, no research work has derived and compared methods of estimating missing values, particularly for rectangular lattice designs. In this study, the Least Square Method (LSM) and the Analysis of Covariance (ANCOVA) method for estimating missing value in rectangular lattice designs, with and without repetitions, were derived and compared based on four (4) statistical criteria: estimated values, standard errors, $\mathrm{p}$-values and coefficients of determination respectively. Results from the comparison between the derived LSM and the ANCOVA methods showed that the estimates of the LSM appeared more approximate and better than the ANCOVA method in terms of their estimated values, standard errors, p-values and coefficients of determination.
\end{abstract}

Keywords: Rectangular Lattice Design, Missing at Random, Coefficient of Determination, Missing Data, Estimated Value

\section{Introduction}

Missing values occur whenever a valid observation is not available for any one of the experimental units. In experimental work, it frequently happens that one or more experimental units are missing from the data or have to be rejected because of conditions outside the control of the researcher due to improper treatment, destruction of the experimental units, loss of the experimental units, illogical data, the inability to measure certain attributes, mishandling of samples, low signal-to-noise ratio, measurement error, non-response or deleted aberrant value which result in loss of information, non-applicability of the standard form of analysis and introduction of a new problem, (Little and Rubin, 2002; Azadeh et al., 2013; Schmitt et al., 2015). In such cases, the most popular and simple method of handling missing data is to ignore either the projects or the attributes with missing observations, but this technique causes the loss of valuable information and therefore may lead to inaccurate cost estimation models Azadeh et al. (2013). Gad and Ahmed (2006) claimed that ignoring the missing values in this case leads to biased inferences.

The methods of handling missing data are directly related to the mechanisms that caused the incompleteness. Generally, these mechanisms fall into the following three classes; Missing Completely at Random (MCAR), Missing
Not At Random (MNAR) and Missing at Random (MAR) Little and Robin (2002). If the fact that the values are missing does not depend on any value for any of the variables, then the values are said to be MCAR, while values are MAR if the probability that values are missing depends only on the observed, but not on the missing values, after controlling for the observed in the experiment and the MNAR can be considered as an intermediate situation between MCAR and MAR Azadeh et al. (2013).

Different methods in handling missing data in statistical experiments have been derived and compared by various authors; Ogbonnaya and Uzochukwu (2013) suggested a procedure for estimating missing data by the least squares technique in the ANCOVA which is based on Yates' procedure for estimation of missing data in ANOVA models. Joseph and Recai (2002) derived and implemented a new EM algorithm for parameter estimation which converges more rapidly than traditional EM algorithms because it does not treat the random effects as "missing data," but integrates them out of the likelihood function analytically. Ajantha and Bhatra (2015) compared the least square approach with the Bayes procedure and remarked that the least square and Bayes estimated values for the parameters are nearly same due to the normality. Lee (2012) found that though both methods result in a similar performance, the maximum likelihood technique has several advantages over multiple imputation (Allison, 2012; 
Enders, 2010; Amanda and Enders, 2009). Dong and Peng (2013) considered the Multiple Imputation (MI), Full Information Maximum Likelihood (FIML) and Expectation-Maximization (EM) algorithm. Under the three missing data conditions, MI, FIML and EM yielded similar estimates and standard errors (SEs). In terms of SE, MLbased methods outperformed MI by providing slightly smaller SEs. Such result is to be expected because MLbased methods do not involve any randomness whereas MI does. From the current literatures reviewed, it appeared that no methods of estimating missing values in simple and triple rectangular lattice designs, with and without repetitions, have been derived and compared, which leads to the motivation of this paper.

Consequently, interest here is to derive and compare the Least Square and the ANCOVA methods of estimating missing values in rectangular lattice designs. The LSM was derived by minimizing the intra-block error sum of squares with respect to the missing plot and solving for the missing plot to obtain the estimate for when one value is missing for each basic design, while the ANCOVA method was derived using the ANCOVA procedure which gives unbiased estimates of both treatment and error sum of square. The comparison was performed on a simple and triple rectangular lattice for 12 treatments in blocks of $3(k=3)$ under a Missing At Random (MAR) assumption with the purpose of determining the preferred out of the two derived methods in estimating missing values given a rectangular lattice designs, with and without repetition.

\section{Methods}

Estimation of one Missing Value in Simple and Triple Rectangular Lattice Designs with and Without Repetitions

\section{Model for Rectangular Lattice Design with and Without Repetitions}

Suppose that we have an experiment of $t=k(k+1)$ treatment in $k+1$ block of $r$ replicate, the observation may be denoted by $Y_{i j k}$ where $i$ denotes the number of treatments $(i=1,2, \ldots, t) ; j$ denotes the number of replicate $(j=1,2, \ldots, r)$ and $k+1=\mathrm{s}$ denotes the number of blocks $(k=1,2, \ldots, s)$.

Model: The model for this design is expressed as:

$Y_{i j k}=\mu+\tau_{i}+\theta_{j}+\rho_{k(j)}+\varepsilon_{i j k}$

Where:

$Y_{i j k}=$ The response (yield) of treatment, $i$, for a particular $j$ replicate and a particular $k$ block.

$\mu=$ Grand mean

$\tau_{i}=$ Effect of treatment $i$

$\theta_{j}=$ Effect of replicate $j$

$\rho_{k(j)}=$ Effect of block $\mathrm{k}$ within replicate $j$

$\varepsilon_{i j k}=$ Random error associated with the response, $Y_{i j k}$, which is normally distributed about a mean zero $(0)$ and constant variance, $\sigma^{2}$.

Table 1: ANOVA table for a $\mathrm{k}(k+1)$ rectangular lattice design without repetitions

\begin{tabular}{llll}
\hline Source of variation & Degrees of freedom & Sum of squares & Mean squares \\
\hline Replicates & $r-1$ & $\frac{\sum R_{j}^{2}}{k(k+1)}-\frac{G^{2}}{r k(k+1)}$ & \\
Treatments (unadj.) & $k^{2}+k-1$ & $\frac{\sum \tau_{i}^{2}}{r}-\frac{G^{2}}{r k(k+1)}$ & \\
Blocks (rep.)) Adj. & $r k$ & $\frac{\sum C_{j k}^{2}}{r(r k-k-1)}-\frac{\sum R_{c}^{2}}{r(k+1)(r k-k-1)}-\frac{\sum S_{k}^{2}}{r(r-1)(k+1)(r k-k-1)}$ & $E_{b}($ error $)$ \\
Intra-block error & $(r-1)\left(k^{2}-1\right)-k$ & By Subtraction & $E_{e}($ error $)$ \\
Total & $r k^{2}+r k-1$ & $\sum Y_{i j k}^{2}-\frac{G^{2}}{r k(k+1)}$ & \\
\hline
\end{tabular}

Table 2: ANOVA table for a $k(k+1)$ rectangular lattice design with repetitions

\begin{tabular}{|c|c|c|c|}
\hline Source of variation & Degrees of freedom & Sum of squares & Mean squares \\
\hline Replicates & $r-1$ & $\frac{\sum_{k(k+1)} R_{j}^{2}}{k(k k(k+1)}-\frac{G^{2}}{r k(k)}$ & \\
\hline Treatments (unadj.) & $k^{2}+k-1$ & $\frac{\sum \tau_{i}^{2}}{r}-\frac{G^{2}}{r k(k+1)}$ & \\
\hline Component (a) & $k(r-n)$ & $\frac{\sum_{h=x}^{y} \sum_{k=1}^{k+1} A_{h k}^{2}}{n k}-\frac{\sum_{i=x}^{v=z} D_{i}^{2}}{n k(k+1)}$ & \\
\hline Component (b) & $n k$ & $\frac{\sum C_{j k}^{2}}{r(n k-k-1)}-\frac{\sum R_{c}^{2}}{r(k+1)(n k-k-1)}-\frac{\sum S_{k}^{2}}{r(n-1)(k+1)(n k-k-1)}$ & $E_{b}$ (error) \\
\hline Intra-block error & $(r-1)\left(k^{2}-1\right)-k$ & By Subtraction & $E_{e}$ (error) \\
\hline Total & $r k^{2}+r k-1$ & $\sum Y_{i j k}^{2}-\frac{G^{2}}{r k(k+1)}$ & \\
\hline
\end{tabular}


The model (1) leads to ANOVA Table 1 and 2 respectively, where $R_{j}$ is the sum of the yields of the treatments for $j^{\text {th }}$ replicate; $\tau_{i}$ is the sum of the yields from all replicates of the treatments $i$; $C_{j k}$ is the total (over all replicates) of all treatments in the block minus (-) $r B_{j k}: r$ is the number of replications; $B_{j k}$ is the sum of the $k^{\text {th }}$ plot in the $k^{\text {th }}$ block of the $j^{\text {th }}$ replicate; $r=n p$ is the number of repetitions; $n$ is the number of times the basic design (simple or triple rectangular lattice design) is replicated; $p$ is the number of repetitions; $R_{c}$ is the sum of the $C_{j k}$ in the $j^{\text {th }}$ replicate; $A_{h k}$ is the difference between blocks within group $h$ of block $k ; \quad \sum_{i=x}^{y} D_{i}^{2}=D_{x}^{2}+D_{y}^{2} \quad$ and $\sum_{i=x}^{z} D_{i}^{2}=D_{x}^{2}+D_{y}^{2}+D_{z}^{2}$ if the design is Simple Rectangular Lattice (SRL) and Triple Rectangular Lattice (TRL) designs respectively such that $D_{x}=R_{x 1}-R_{x 2}, D_{y}=R_{y 1}-R_{y 2}$ and $D_{z}=R_{z 1^{-}} R_{z 2}$ are the differences between the repetitions of group $X, Y$ for SRL and $X, Y$ and $Z$ for TRL designs respectively; $E_{b}$ is the block error; $E_{e}$ is the intra-block error; $S_{k}$ is the sum of the same peer in the same row; $G$ is the grand total; $k$ is the block size.

Estimation of Missing Value in Rectangular Lattice Designs without Repetitions for One Observation

Suppose the yield on the plot in replicate $j$, block $k$ and receiving treatment $i$ denoted by $u$ is missing. Let $R_{j}$, $\tau_{i}, C_{j k}, R_{c}, S$ and $G$ retain their usual meanings so that $R_{j}^{\prime}$ is the sum of the observations of all the treatments in replicate $j ; \tau_{i}^{\prime}$ is the sum of the observations from all replicates of treatment $i$; $C_{j k}^{\prime}$ is the total (over replicate $\mathrm{j}$ ) of all treatments in block k minus $(-) r B_{j k}^{\prime} ; R_{c}^{\prime}$ is the sum of the $C_{j k}$ in replicate $j ; S_{k}^{\prime}$ is the sum of the observations in the same row and $G^{\prime}$ is the unknown grand total. This estimate is the same for the Simple Rectangular Lattice (SRL) and Triple Rectangular Lattice (TRL) designs except for the value of $r$ which is 2 for the SRL and 3 for the TRL designs:

$$
\begin{aligned}
& S S_{E}=\left(u^{2}+\sum Y_{j k k}^{2}-\frac{\left(G^{\prime}+u\right)^{2}}{B}\right)-\left(\frac{\left(R_{j}^{\prime}+u\right)^{2}}{A}+\frac{\sum R_{j}^{2}}{A}-\frac{\left(G^{\prime}+u\right)^{2}}{B}\right) \\
& -\left(\frac{\left(\tau_{i}^{\prime}+u\right)^{2}}{r}+\frac{\sum \tau_{i}^{2}}{r}-\frac{\left(G^{\prime}+u\right)^{2}}{B}\right) \\
& -\left(\frac{\left(C_{j k}^{\prime}+u\right)^{2}}{C}+\frac{\sum C_{j k}^{2}}{C}-\frac{\left(R_{c}^{\prime}+u\right)^{2}}{D}+\frac{\sum R_{c}^{2}}{D}-\frac{\left(S_{k}^{\prime}+u\right)^{2}}{E}+\frac{\sum S_{k}^{2}}{E}\right)
\end{aligned}
$$

Then we minimize the intra-block error sum of squares (2) with respect to the missing plot, $u$, say $Q$, we have:

$$
\begin{aligned}
& \frac{\partial Q}{\partial u}=2 u+\frac{2\left(G^{\prime}+u\right)}{B}-\frac{2\left(R_{j}^{\prime}+u\right)}{A}-\frac{2\left(\tau_{i}^{\prime}+u\right)}{r} \\
& -\frac{2\left(C_{j k}^{\prime}+u\right)}{C}+\frac{2\left(R_{c}^{\prime}+u\right)}{D}+\frac{2\left(S_{k}^{\prime}+u\right)}{E}=0
\end{aligned}
$$

Then solving for $u$, we have:

$$
\begin{aligned}
& F(r-1)\left[r R_{j}^{\prime}+A \tau_{i}^{\prime}-G^{\prime}\right] \\
u= & \frac{+k\left[(r-1)(k+1) C_{j k}^{\prime}-(r-1) R_{c}^{\prime}-S_{k}^{\prime}\right]}{F\left[(r-1)^{2}\left(k^{2}+k-1\right)-k\right]}
\end{aligned}
$$

where, $A=k(k+1), B=r k(k+1), C=r(r k-k-1), D=r(k$ $+1)(r k-k-1), E=r(r-1)(k+1)(r k-k-1), F=(r k-k-1)$.

Therefore, for simple rectangular lattice designs $(r=$ 2 ) and for triple rectangular lattice designs $(r=3)$ :

$$
\begin{aligned}
& F(r-1)\left[r R_{j}^{\prime}+A \tau_{i}^{\prime}-G^{\prime}\right] \\
u= & \frac{+k\left[(r-1)(k+1) C_{j k}^{\prime}-(r-1) R_{c}^{\prime}-S_{k}^{\prime}\right]}{F\left[(r-1)^{2}\left(k^{2}+k-1\right)-k\right]}
\end{aligned}
$$

\section{Estimation of Missing Value in Rectangular Lattice} Designs with Repetitions for One Observation

Suppose the yield on the plot in replicate $j$, block $k$ and receiving treatment $i$ denoted by $u$ is missing. Let $R_{j}, \tau_{i}, C_{j k}$, $R_{c}, S$ and $G$ retain their usual meanings so that $R_{j}^{\prime}$ is the sum of the observations of all the treatments in replicate $j$; $\tau_{i}^{\prime}$ is the sum of the observations from all replicates of treatment $i ; C_{j k}^{\prime}$ is the total (over replicate $j$ ) of all treatments in block $k$ minus $(-) r B_{j k}^{\prime} ; R^{\prime}$ is the sum of the $C_{j k}$ in replicate $j ; S^{\prime}$ is the sum of the observations in the same row and $G^{\prime}$ is the unknown grand total. The estimate, $\mathrm{u}$ for one observation missing is the same for both the SRL and TRL designs except for the values of $F^{\prime}$ which is $D_{x}+$ $D_{y}$ for SRL and $D_{x}+D_{y}+D_{z}$ for TRL designs for all cases:

$$
\begin{aligned}
& S S_{E}=\left(u^{2}+\sum Y_{i j k}^{2}-\frac{\left(G^{\prime}+u\right)^{2}}{B}\right)-\left(\frac{\left(R_{j}^{\prime}+u\right)^{2}}{A}+\frac{\sum R_{j}^{2}}{A}-\frac{\left(G^{\prime}+u\right)^{2}}{B}\right) \\
& -\left(\frac{\left(\tau_{i}^{\prime}+u\right)^{2}}{r}+\frac{\sum \tau_{i}^{2}}{r}-\frac{\left(G^{\prime}+u\right)^{2}}{B}\right) \\
& -\left(\frac{\left(A_{h k}+u\right)^{2}}{n k}+\sum_{h=x}^{y} \sum_{k=1}^{k+1} \frac{A_{h k}^{2}}{n k}-\frac{\left(\sum_{i=x}^{v} D_{i}+u\right)^{2}}{n A}+\frac{\left(\sum_{i=x}^{v} D_{i}^{2}\right.}{n A}\right) \\
& -\left(\frac{\left(C_{j k}^{\prime}+u\right)^{2}}{C}+\frac{\sum C_{j k}^{2}}{C}-\frac{\left(R_{c}^{\prime}+u\right)^{2}}{D}+\frac{\sum R_{c}^{2}}{D}-\frac{\left(S_{k}^{\prime}+u\right)^{2}}{E}+\frac{\sum S_{k}^{2}}{E}\right)
\end{aligned}
$$


Minimizing the intra-block error sum of squares (6) with respect to the missing plot, $u$, say $Q$ for the we have:

$$
\begin{aligned}
& \frac{\partial Q}{\partial u}=2 u+\frac{2\left(G^{\prime}+u\right)}{B}-\frac{2\left(R_{j}^{\prime}+u\right)}{A} \\
& -\frac{2\left(\tau_{i}^{\prime}+u\right)}{r}-\frac{2\left(A_{h k}+u\right)}{n k}+\frac{2\left(\sum_{i=x}^{y, z} D_{i}+u\right)}{n A}-\frac{2\left(C_{j k}^{\prime}+u\right)}{C} \\
& +\frac{2\left(R_{c}^{\prime}+u\right)}{D}+\frac{2\left(S_{k}^{\prime}+u\right)}{E}=0
\end{aligned}
$$

Then solving for $u$, we have:

$$
\begin{gathered}
F(n-1)\left[n r R_{j}^{\prime}+n A \tau_{i}^{\prime}-n G^{\prime}+r(k+1) A_{h k}-r F^{\prime}\right] \\
u=\frac{+n k\left[(n-1)(k+1) C_{j k}^{\prime}-(n-1) R_{c}^{\prime}-S_{k}^{\prime}\right]}{F\left[n(n-1)(r-1)\left(k^{2}+k-1\right)-k(r n-r-n)\right]}
\end{gathered}
$$

where, $A=k(k+1), B=r k(k+1), C=r(n k-k-1), D=r(k$ $+1)(n k-k-1), E=r(n-1)(k+1)(n k-k-1), F=(n k-k-1)$ and $F^{\prime}=\sum_{i=x}^{y, z} D_{i}^{2}$ depends on whether is a SRL or TRL design respectively.

For simple rectangular lattice designs $(n=2)$ :

$$
\begin{aligned}
& F(n-1)\left[n r R_{j}^{\prime}+n A \tau_{i}^{\prime}-n G^{\prime}+r(k+1) A_{h k}-r\left(D_{x}+D_{y}\right)\right] \\
& u=+n k\left[(n-1)(k+1) C_{j k}^{\prime}-(n-1) R_{c}^{\prime}-S_{k}^{\prime}\right] \\
& F\left[n(n-1)(r-1)\left(k^{2}+k-1\right)-k(r n-r-n)\right]
\end{aligned}
$$

for triple rectangular lattice designs $(n=3)$ :

$$
\begin{gathered}
F(n-1)\left[\begin{array}{l}
n r R_{j}^{\prime}+n A \tau_{i}^{\prime}-n G^{\prime} \\
+r(k+1) A_{h k}-r\left(D_{x}+D_{y}+D_{z}\right)
\end{array}\right] \\
u=\frac{+n k\left[(n-1)(k+1) C_{j k}^{\prime}-(n-1) R_{c}^{\prime}-S_{k}^{\prime}\right]}{F\left[n(n-1)(r-1)\left(k^{2}+k-1\right)-k(r n-r-n)\right]}
\end{gathered}
$$

\section{Analysis of Covariance (ANCOVA) Method}

In the application of the analysis of covariance (ANCOVA) technique to one missing observation, we let $n$ equal the total number of observations in the experiment including the missing value. Also consider the original data as the dependent variable $Y$ of the covariance analysis and insert the value of zero in the cell which has the missing observation. Then set up a concomitant variable $X$ which takes the value $-n$ in the cell corresponding to the substituted zero value and the value of zero elsewhere. The usual computational procedures of the covariance analysis automatically provides unbiased test of significance and the multiple of the total number of observations with the partial error regression coefficient gives the estimate of the missing value. However, estimates of the functions of the dependent variable data, such as treatment means, must be adjusted to the value of zero for the concomitant variable rather than to the observed average value (grand average) of the concomitant variable.

From the description of the ANCOVA method of estimating missing value, the procedure which gives unbiased estimates of both treatment and error sum of square can be simplified as follows:

1. Set $Y=0$ for the missing observation

2. Define a covariate as $X=0$ for an unobserved $Y$ and $X=-n$ for $Y=0$

3. Carry out the analysis of covariance

4. Compute $\hat{\beta}_{E}=E_{x y} / E_{x x}$ and multiply by $\mathrm{n}$ to estimate the missing value. where $E_{x x}$ is the error sum of squares and product for the concomitant variable $x$; $E_{x y}$ is the error sum of squares and product for the concomitant variable $\mathrm{X}$ and the dependent variable $Y$; and $\hat{\beta}_{E}$ is the unbiased estimate for the missing value

\section{Standard Errors}

The standard error of mean estimates the variability between sample means that can be obtained if multiple samples are taken from the same population. In other words, standard error is a measure of statistical accuracy of an estimate observed by taking the square root of the error variance of the difference between the treatment means.

\section{Standard Errors for Rectangular Lattice Designs}

In simple and triple rectangular lattice designs with and without repetitions, there are two cases in which the treatment means can be adjusted given missing values. The expressions of the standard error for these cases are the same for SRL and TRL designs with and without repetitions respectively, except for the values of $r$, where $r$ is four (4) for SRL and six (6) for TRL designs with repetitions. While $r$ is two (2) for SRL and three (3) for TRL without repetitions.

The standard error of the difference between the means of two treatments occurring together in the same block given missing data is:

$$
S E_{1}=\sqrt{E_{e}\left(\frac{k+1}{k}\right)\left\{\frac{2}{r}+\frac{k(k+1)}{r\left((r-1)\left(k^{2}-1\right)-k\right)}\right\}}
$$


The standard error of the difference between the means of two treatments not occurring together in the same block given missing data is:

$$
S E_{2}=\sqrt{E_{e}\left(\frac{k+2}{k}\right)\left\{\frac{2}{r}+\frac{k^{3}}{r(k+2)\left((r-1)\left(k^{2}-1\right)-k\right)}\right\}}
$$

\section{Standard Error for the Analysis of Covariance (ANCOVA) Method}

In the Analysis of Covariance method, the standard error needed to measure of statistical accuracy of an estimate is given as:

$$
S E=\sqrt{\sigma^{2}\left(\frac{n^{2}}{E_{x x}}-1\right)}
$$

where, $\sigma^{2}$ is estimated by $s^{2}$ which is the residual sum of square resulting from routine application of the analysis of covariance.

\section{Numerical Illustration}

Analysis of Simple and Triple Rectangular Lattice Designs Without Replications and with Repetitions Respectively

For the purpose of numerical illustration, we adopted a rectangular lattice example from Cox and Cochran (1957, page 417-418) as set up in Table 3. The data are on a triple triangular lattice for 12 treatments in blocks of $3(k=3)$. Artificial data were assembled by taking true treatment effects and adding to them true block effects. For the case of Rectangular lattice designs without repetitions, $r=2, k=3$ for SRL and $r=3, k=3$ for TRL designs respectively. Using a rectangular lattice example from Harshbarger (1949), the data are on 12 treatments of a $3 * 4$ rectangular lattice with repetitions. The experiment was set up as in Table 4 where $n=2, n=4$ for SRL design and $n=3, n=6$ for TRL design, $k=3$ and $k+1=4$ are the same for both the SRL and TRL designs.

\section{Results}

Estimation of Missing Value Rectangular Lattice Designs with and Without Repetitions Using the Least Square Method (LSM).

Suppose that the observation 16 for treatment (1) in block $X 1$ of replicate $\mathrm{X}$ of the presented rectangular lattice design without repetition had been missing in Table 3 and suppose that the observation 13.06 for treatment (1) in block1 of replicate 1 of the presented rectangular lattice design with repetition had been missing in Table 4, using (5) and (8) the estimated values for the simple and triple rectangular lattice designs, with and without repetitions were obtained respectively, while the actual and the estimated standard errors for the simple and triple rectangular lattice designs, with and without repetitions were obtained using (11) and (12) respectively as shown in Table 5 .

Table 5 showed the results for SRL and TRL designs with and without repetitions. The decrease in standard error and p-value for TRL design was as a result of increase in repetitions which increased the precision of the estimated values. Also the estimated values for SRL and TRL designs with repetitions gave a better approximation than without repetitions and the estimated coefficients of determination for the SRL and TRL designs are approximate to the actual coefficients of determination which indicates the effectiveness of the proposed procedures.

Table 3: Plan and Observations for a $3 * 4$ Rectangular Lattice: (treatment numbers are enclosed in parentheses)

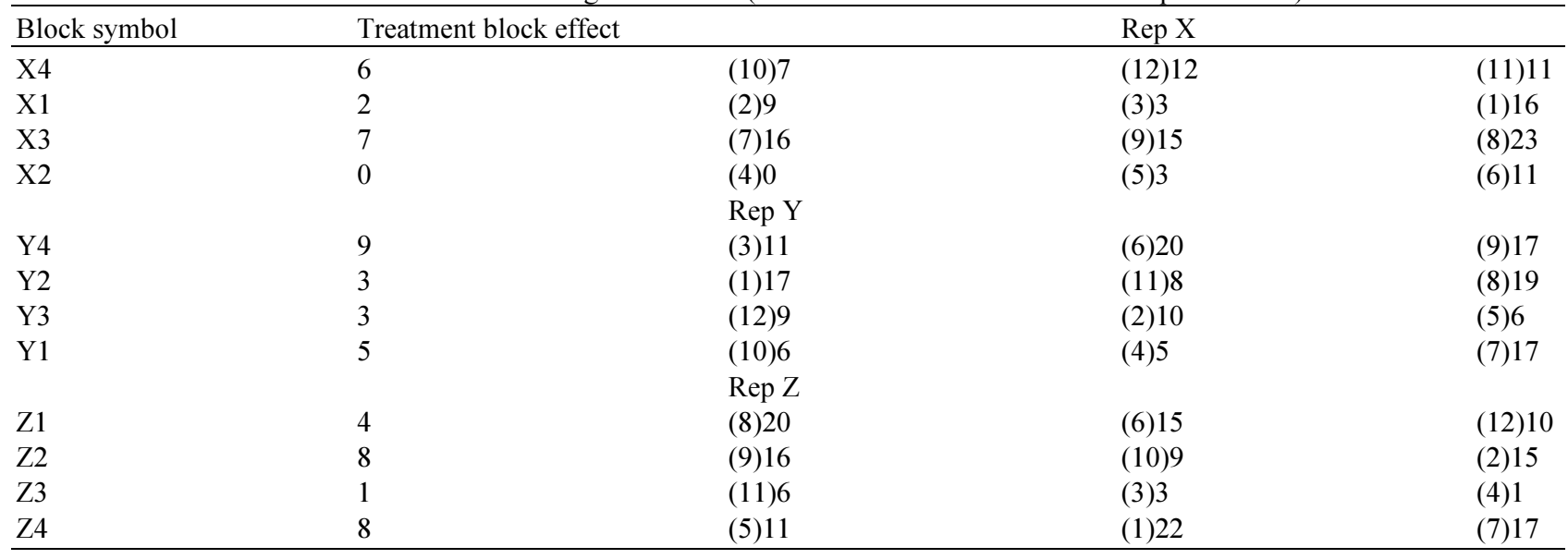


Table 4: Yield of Alfalfa for a $3 * 4$ rectangular lattice design (Treatment numbers are enclosed in parentheses)

\begin{tabular}{|c|c|c|c|c|c|c|c|c|c|}
\hline \multirow{2}{*}{$\frac{\text { Blocks }}{\text { Group X }}$} & \multicolumn{3}{|c|}{ Repetition 1} & \multirow[t]{2}{*}{$\begin{array}{l}\text { Block } \\
\text { totals }\end{array}$} & \multirow[t]{2}{*}{ Blocks } & \multicolumn{3}{|c|}{ Repetition 2} & \multirow[t]{2}{*}{$\begin{array}{l}\text { Block } \\
\text { totals }\end{array}$} \\
\hline & & & & & & & & & \\
\hline 1 & (1)13.06 & (2)5.68 & (3) 6.28 & 25.02 & 1 & (1) 10.70 & (2)4.36 & (3)5.66 & 20.72 \\
\hline 2 & (4)8.24 & (5)8.32 & (6)7.84 & 24.40 & 2 & (4)10.34 & (5)6.44 & (6)10.06 & 26.84 \\
\hline 3 & (7)7.32 & (8)6.86 & (9)5.04 & 19.22 & 3 & (7)5.62 & (8)7.90 & (9)7.70 & 21.22 \\
\hline 4 & (10)8.88 & (11)11.42 & (12)10.38 & 30.68 & 4 & (10)6.46 & (11)8.36 & (12)6.74 & 21.56 \\
\hline Total & & & $\mathrm{R} \times 1$ & 99.32 & & & & $\mathrm{R} \times 2$ & 90.34 \\
\hline \multicolumn{10}{|l|}{ Group Y } \\
\hline 1 & (4) 8.55 & (7)9.72 & (10)4 & 22.27 & 1 & (4) 10.74 & (7) 8.18 & (10)8.92 & 27.84 \\
\hline 2 & (1) 10.56 & (8) 6.60 & (11)9.64 & 26.80 & 2 & (1) 12.62 & (8)8.52 & (11)11.92 & 33.06 \\
\hline 3 & (2) 6.76 & (5) 8.6 & (12)8.06 & 23.42 & 3 & (2)9.18 & (5)9.76 & (12)8.34 & 27.28 \\
\hline 4 & (3)7.6 & (6)7.82 & (9)7.98 & 23.40 & 4 & (3) 7.76 & (6) 10.38 & (9) 10.70 & 28.84 \\
\hline Total & & & Ry1 & 95.89 & & & & Ry2 & 117.02 \\
\hline \multicolumn{10}{|l|}{ Group Z } \\
\hline 1 & (5)9.86 & (9)9.28 & (11)13.04 & 32.18 & 1 & $(5) 5.68$ & (9)9.40 & (11)9.98 & 28.06 \\
\hline 2 & (3)8.74 & (7)9.34 & (12) 10.68 & 28.76 & 2 & (3)5.46 & $(7) 9.41$ & (12) 10.52 & 25.39 \\
\hline 3 & (1)11.36 & (6)8.52 & $(10) 6.32$ & 26.20 & 3 & (1) 14.02 & (6) 11.76 & (10)8.84 & 34.62 \\
\hline 4 & (2)5.54 & (4) 10.58 & (8) 8.88 & 25.00 & 4 & (2) 8.96 & (4) 12 & (8)9.64 & 30.60 \\
\hline Total & & & Rz1 & 112.14 & & & & Rz2 & 118.67 \\
\hline
\end{tabular}

Table 5: Estimates for simple and triple rectangular lattice designs without repetitions and with repetitions respectively

\begin{tabular}{|c|c|c|c|c|c|}
\hline & & \multicolumn{2}{|l|}{ SRL } & \multicolumn{2}{|l|}{ TRL } \\
\hline & & Actual & Estimated & Actual & Estimated \\
\hline \multirow[t]{5}{*}{ Without repetitions } & Value & 16 & 9.75 & 16 & 19.5100 \\
\hline & $\mathrm{SE}_{1}$ & 0 & 8.0270 & 0 & 0.8059 \\
\hline & $\mathrm{SE}_{2}$ & 0 & 7.5087 & 0 & 0.8191 \\
\hline & P-value & - & 0.1869 & - & 0.0001 \\
\hline & $R^{2}$ & 1.00 & 0.894 & 1.00 & 0.9950 \\
\hline \multirow{5}{*}{ With repetitions } & Value & 13.06 & 13.02 & 13.06 & 11.5400 \\
\hline & $\mathrm{SE}_{1}$ & 1.3337 & 1.0126 & 0.6713 & 0.3380 \\
\hline & $\mathrm{SE}_{2}$ & 1.3835 & 1.0608 & 0.6946 & 0.3846 \\
\hline & P-value & 0.0001 & 0.0001 & 0.0001 & 0.0001 \\
\hline & $R^{2}$ & 0.902 & 0.912 & 0.966 & 0.9700 \\
\hline
\end{tabular}

Table 6: ANCOVA for Simple and Triple rectangular lattice designs with and without repetitions respectively

\begin{tabular}{|c|c|c|c|c|c|}
\hline & & \multicolumn{2}{|l|}{ SRL } & \multicolumn{2}{|l|}{ TRL } \\
\hline & & Actual & Estimated & Actual & Estimated \\
\hline \multicolumn{6}{|c|}{ Without replications } \\
\hline & Value & 16 & 5.9100 & 16 & 6.0900 \\
\hline & SE & 0 & 1.8002 & 0 & 2.1853 \\
\hline & P-value & - & 0.4378 & - & 0.6834 \\
\hline & $R^{2}$ & - & 0.0230 & - & 0.0220 \\
\hline \multirow[t]{4}{*}{ With repetitions } & Value & 13.06 & 11.0600 & 13.06 & 10.7600 \\
\hline & $\mathrm{SE}$ & 0 & 1.8890 & 0 & 1.3816 \\
\hline & P-value & - & 0.1272 & - & 0.0001 \\
\hline & $R^{2}$ & - & 0.0240 & - & 0.1230 \\
\hline
\end{tabular}

Estimation of Missing value Rectangular Lattice Designs with and Without Repetitions using ANCOVA Method

Suppose that the observation 16 for treatment (1) in block $X 1$ of replicate $X$ of the presented rectangular lattice design without repetition had been missing in Table 3 and suppose that the observation 13.06 for treatment (1) in block 1 of replicate 1 of the presented rectangular lattice design with repetition had been missing in Table 4, the Analysis of covariance method in (2.2) was adopted following the underlying procedures, the results were presented in Table 6 . The estimated values for the simple and triple rectangular lattice designs, with and without repetitions were obtained by following the ANCOVA procedures in (2.2), while the actual and the estimated standard errors for the simple and triple rectangular lattice designs, with and without repetitions were obtained using (13) respectively. 
Table 7: Comparison of the Least Square Method (LSM) and the ANCOVA method of estimating missing values in rectangular lattice design, with and without repetitions

\begin{tabular}{|c|c|c|c|c|}
\hline & & Values & LSM & ANCOVA \\
\hline \multirow[t]{10}{*}{ Without repetitions } & \multirow[t]{5}{*}{ SRL } & Actual & 16 & 16.0000 \\
\hline & & Estimated & 9.75 & 5.9100 \\
\hline & & SE & $8.0270,7.5087$ & 1.8002 \\
\hline & & P-value & 0.1869 & 0.4378 \\
\hline & & $R^{2}$ & 0.995 & 0.0230 \\
\hline & \multirow[t]{5}{*}{ TRL } & Actual & 16 & 16.0000 \\
\hline & & Estimated & 19.51 & 6.0900 \\
\hline & & SE & $0.8059,0.8191$ & 2.1853 \\
\hline & & P-value & 0.0001 & 0.6834 \\
\hline & & $R^{2}$ & 0.995 & 0.0220 \\
\hline \multirow[t]{10}{*}{ With repetitions } & \multirow[t]{5}{*}{ SRL } & Actual & 13.06 & 13.0600 \\
\hline & & Estimated & 13.02 & 11.0600 \\
\hline & & SE & $1.0126,1.0608$ & 1.8890 \\
\hline & & P-value & 0.0001 & 0.1272 \\
\hline & & $R^{2}$ & 0.912 & 0.0240 \\
\hline & \multirow[t]{5}{*}{ TRL } & Actual & 13.06 & 13.0600 \\
\hline & & Estimated & 11.54 & 10.7600 \\
\hline & & SE & $0.3380,0.3846$ & 1.3816 \\
\hline & & P-value & 0.0001 & 0.0001 \\
\hline & & $R^{2}$ & 0.970 & 0.1230 \\
\hline
\end{tabular}

Table 6 ANCOVA result showed that theSRL and TRL designs with repetitions gave a better approximation than without repetitions in terms of the four evaluation criteria.

Table 7 showed the results of the comparison between the LSM and ANCOVA method. It was found that the least square method gave a closer and better approximation than the ANCOVA method in terms of their estimated values, standard errors, p-values and coefficients of determination for both the simple and triple rectangular lattice designs, with and without repetition.

\section{Discussion}

In this study, the least square and the ANCOVA methods have been derived for the estimation of missing value in simple and triple rectangular lattice designs (with and without repetitions) when one observation is missing using the intra-block information. The Least Square method was compared with that of ANCOVA method. From the least square estimates in Table 7, the estimated values $(13.02,11.54)$, standard errors $(8.0270$, $7.5087 ; 0.8059,0.8191)$, p-value $(0.1869,0.0001)$ and coefficients of determination $(0.894,0.995)$ for simple and triple rectangular lattice design without repetitions were obtained; and estimated values $(9.75,19.51)$, standard errors (1.0126, 1.0608; 0.3380, 0.3846), p-value $(0.0001,0.0001)$ and coefficients of determination $(0.912,0.970)$ for simple and triple rectangular lattice design with repetitions were also obtained. While from the ANCOVA estimates, the estimated values (5.91, $6.09)$, standard errors $(1.8002 ; 2.1853)$, p-value $(0.4378$, $0.6834)$ and coefficients of determination $(0.023,0.022)$ for simple and triple rectangular lattice design without repetitions were obtained; and estimated values (11.06, 10.76), standard errors $(1.8890 ; 1.3816)$, p-value $(0.1272,0.0001)$ and coefficients of determination $(0.024,0.123)$ for simple and triple rectangular lattice design with repetitions were also obtained respectively, which showed that the least square method gave a closer and better approximation than the ANCOVA method in terms of their estimated values, standard errors,p-values and coefficients of determination.

\section{Conclusion}

From the results and discussion above, it was found that to increase the precision of the estimates, the experiment should either be replicated or repeated since the estimates of the designs with higher replications and those with repetitions appeared to be more approximate. From the comparison between the least square method and the ANCOVA method, the least square method appeared better than the ANCOVA method in terms of their estimated values, standard errors, p-values and coefficients of determination. And it could be recommend that in performing a large experiment which involves the use rectangular lattice designs (simple or triple), the derived least square procedures in this study should be adopted. And to increase the precision of the estimates, the basic design under consideration should be replicated or repeated.

\section{Acknowledgement}

The authors would like to thank the anonymous referees for their careful reading of our manuscript and helpful suggestions. 


\section{Author's Contributions}

All the authors contributed equally to writing the manuscript and they had an equal say in reviewing and approving the final version.

\section{Ethics}

This article is original. The corresponding author confirms that all of the other authors have read and approved the manuscript and there are no ethical issues involved.

\section{References}

Ajantha, R. and C. Bhatra, 2015. Bayesian estimation for missing values in Latin square design. Int. J. Math. Stat. Invent., 3: 6-8.

Allison, P.D., 2012. Handling missing data by maximum likelihood. Haverdford, PA: SAS Global Forum.

Amanda, N.B. and C.K. Enders, 2009. An introduction to modern missing data analyses. J. School Psychol., 48: 5-37. DOI: 10.1016/j.jsp.2009.10.001

Azadeh, A., S.M. Asadzadeh, R. Jafari-Marandi, S. Nasari-Shirkouhi and K.G. Baharian et al., 2013. Optimum estimation of missing values in randomized complete block design by genetic algorithm. Knowl. Based Syst., 37: 37-47. DOI: $10.1016 /$ j.knosys.2012.06.014

Cox, G.M. and W.G. Cochran, 1957. Experimental designs. Wiley, London.

Dong, Y. and C.J. Peng, 2013. Principled missing data methods for researchers. Springer Open J., 2: 222-222. DOI: $10.1186 / 2193-1801-2-222$
Enders, C.K., 2010. Applied Missing Data Analysis. 1st Edn., Guilford Press, New York, ISBN-10: 1606236393 , pp: 377.

Gad, A.M. and A.S. Ahmed, 2006. Analysis of longitudinal data with intermittent missing values using the stochastic EM algorithm. Comput. Stat. Data Anal., 50: 2702-2714. DOI: 10.1016/j.csda.2005.04.006

Harshbarger, B., 1949. Triple rectangular lattices. Biometrics, 5: 1-13. DOI: $10.2307 / 3001888$

Joseph, L.S. and M.Y. Recai, 2002. Computational strategies for multivariate linear mixed-effects models with missing values. J. Comput. Graphical Stat., 11: 37-457.

DOI: $10.1198 / 106186002760180608$

Lee, I.H., 2012. Strategies for handling missing data with maximum likelihood estimation in career and technical education research. Career Tech. Educ. Res., 37: 297-310. DOI: 10.5328/cter37.3.297

Little, R.J.A. and D.B. Rubin, 2002. Statistical Analysis with Missing Data. 2nd Edn., Wiley, New York, ISBN-10: 0471183865, pp: 408.

Ogbonnaya, C.E. and E.C. Uzochukwu, 2013. Estimation of missing data in the analysis of covariance: A least-squares approach. Commun. Stat.-Theory Meth., 45: 1902-1909. DOI: $10.1080 / 03610926.2013 .868000$

Schmitt, P., J. Mandel and M. Guedj, 2015. A comparison of six methods for missing data imputation. J. Biometr. Biostat., 6: 224-224. DOI: $10.4172 / 2155-6180.1000224$ 